\title{
Channel noise-induced phase transition of spiral wave in networks of Hodgkin-Huxley neurons
}

\author{
MA Jun ${ }^{1,2 *}$, WU Ying ${ }^{3}$, YING HePing ${ }^{4} \& \mathrm{JIA} \mathrm{Ya}^{2}$ \\ ${ }^{1}$ Department of Physics, Lanzhou University of Technology, Lanzhou 730050, China; \\ ${ }^{2}$ Department of Physics, Huazhong Normal University, Wuhan 430079, China; \\ ${ }^{3}$ School of Science, Xi'an University of Technology, Xi'an 710048, China; \\ ${ }^{4}$ Zhejiang Institute of Modern Physics, Zhejiang University, Hangzhou 310027, China
}

Received July 19, 2010; accepted September 21, 2010

\begin{abstract}
The phase transition of spiral waves in networks of Hodgkin-Huxley neurons induced by channel noise is investigated in detail. All neurons in the networks are coupled with small-world connections, and the results are compared with the case for regular networks, in which all neurons are completely coupled with nearest-neighbor connections. A statistical variable is defined to study the collective behavior and phase transition of the spiral wave due to the channel noise and topology of the network. The effect of small-world connection networks is described by local regular networks and long-range connection with certain probability $p$. The numerical results confirm that (1) a stable rotating spiral wave can be developed and maintain robust with low $p$, where the breakup of the spiral wave and turbulence result from increasing the probability $p$ to a certain threshold; (2) appropriate intensity of the optimized channel noise can develop a spiral wave among turbulent states in small-world connection networks of $\mathrm{H}-\mathrm{H}$ neurons; and (3) regular connection networks are more robust to channel noise than small-world connection networks. A spiral wave in a small-world network encounters instability more easily as the membrane temperature is increased to a certain high threshold.
\end{abstract}

breakup, channel noise, factor of synchronization, probability of long-range connection

Citation: $\quad$ Ma J, Wu Y, Ying H P, et al. Channel noise-induced phase transition of spiral wave in networks of Hodgkin-Huxley neurons. Chinese Sci Bull, 2011, 56: 151-157, doi: 10.1007/s11434-010-4281-2

Collective electrical behaviors of neurons and oscillators in networks often have spatiotemporal patterns [1-11]. A spiral wave is one such spatial pattern and is often observed in excitable and oscillatory media. In experimental studies, most of the works used to study the chemical wave in the Belousov-Zhabotinsky reaction [12], and many other theoretical and numerical works on spiral waves have been reported [13-18]. The importance of studying spiral waves is that it gives important clues as to how to remove spiral waves in cardiac tissue and prevent ventricular fibrillation [19] and allows a better understanding of the nonlinear dynamics from a spiral wave to turbulence. There is evidence that a spiral wave in cardiac tissue is harmful, and thus,

*Corresponding author (email: hyperchaos@163.com) many effective schemes have been proposed to eliminate spiral waves in media. For example, the scheme of periodical forcing is proposed to eliminate the spiral waves and turbulence by generating a target wave or travelling wave in the media [20,21]. Transition from a spiral wave to other states induced by a polarized field [22], deformation of media $[23,24]$, and the synchronization of spiral waves [25] have also been investigated extensively. In particular, the noise-induced formation and development of spiral waves in a reaction-diffusion system was discussed by Hou and Xin in detail [26]. The dynamics of spiral waves and control pattern selection in reaction-diffusion systems have been studied extensively while few works have been reported on the development and phase transition of the spiral wave in the networks of neurons, and its role in signal communica- 
tion in the networks is unknown. A neuronal system consists of a large number of neurons [27] with complex connections. Normal electric activity of neurons is critical for signal communication among neurons. Stable rotating spiral waves in rat neocortical slices visualized by voltagesensitive dye imaging were found in experiments [6,7]. As noted in [6,7], spiral waves might serve as pacemakers for the emergent population to generate periodic activity in a nonoscillatory network without the need for individual cellular pacemakers. It is interesting to simulate and investigate the formation and breakup of spiral waves in networks of neurons with different topologies. Perc et al. made great progress in the formation of spiral waves in networks [8-10,28,29]. He et al. [30] presented excellent results on the formation of spiral waves in small-world networks of FitzHugh-Nagumo neurons and confirmed that the destructive effect of an inhomogeneous medium on spiral waves can be decreased or removed by appropriate small-world connections. Some aspects of this topic remain unclear; for example, the dynamics of spiral waves in small-world networks of neurons and the effects of channel noise and the size of the network on a spiral wave. It is better to study spiral wave dynamics in networks of Hodgkin-Huxley $(\mathrm{H}-\mathrm{H})$ neurons than in networks of Hindmarsh-Rose $(\mathrm{H}-\mathrm{R})$ neurons $[4,5]$ because the $\mathrm{H}-\mathrm{R}$ neuron model is a simplified version of the realistic $\mathrm{H}-\mathrm{H}$ model. Channel noise can change the dynamics of $\mathrm{H}-\mathrm{H}$ neurons greatly $[31,32]$. White et al. [31] pointed out that the probabilistic gating of voltage-dependent ion channels is a source of electrical 'channel noise' in neurons. Schmid et al. [32] reported the capacitance fluctuations reducing channel noise in stochastic $\mathrm{H}-\mathrm{H}$ systems. Fox et al. [33] presented the autocorrelation functions of channel noise to estimate the effect of channel noise. The present work investigates the robustness and breakup of a spiral wave in small-world networks of $\mathrm{H}-\mathrm{H}$ neurons in the presence of channel noise. A statistical variable is defined to study the phase transition of spiral waves, and the results are compared with those for regular networks. The external forcing current at all sites (neurons) is set at zero, which makes each single $\mathrm{H}-\mathrm{H}$ neuron quiescent. It is found that appropriate channel noise actively develops spiral waves and maintains its robustness so that signal communication still can pass through these quiescent areas.

\section{Mathematical model and discussion}

The $\mathrm{H}-\mathrm{H}$ neuron mode is more realistic than other presented neuron models. Small-world networks of $\mathrm{H}-\mathrm{H}$ neurons are described as follows:

$$
\begin{aligned}
C_{m} \frac{\mathrm{d} V_{i j}}{\mathrm{~d} t}= & \tilde{g}_{\mathrm{K}} n_{i j}^{4}\left(V_{\mathrm{K}}-V_{i j}\right)+\tilde{g}_{\mathrm{Na}} m_{i j}^{3} h_{i j}\left(V_{\mathrm{Na}}-V_{i j}\right) \\
& +\tilde{g}_{\mathrm{L}}\left(V_{\mathrm{L}}-V_{i j}\right)+I_{i j}+D \sum_{k l} \varepsilon_{i j k l}\left(V_{k l}-V_{i j}\right),
\end{aligned}
$$

$$
\begin{gathered}
\frac{\mathrm{d} m_{i j}}{\mathrm{~d} t}=\alpha_{m}\left(V_{i j}\right)\left(1-m_{i j}\right)-\beta_{m}\left(V_{i j}\right) m_{i j}+\xi_{m}(t), \\
\frac{\mathrm{d} h_{i j}}{\mathrm{~d} t}=\alpha_{h}\left(V_{i j}\right)\left(1-h_{i j}\right)-\beta_{m}\left(V_{i j}\right) h_{i j}+\xi_{h}(t), \\
\frac{\mathrm{d} n_{i j}}{\mathrm{~d} t}=\alpha_{m}\left(V_{i j}\right)\left(1-n_{i j}\right)-\beta_{m}\left(V_{i j}\right) n_{i j}+\xi_{n}(t), \\
\alpha_{m}=\frac{0.1\left(V_{i j}+40\right) \Phi(T)}{1-\exp \left(-\left(V_{i j}+40\right) / 10\right)}, \\
\beta_{m}=4 \Phi(T) \exp \left(-\left(V_{i j}+65\right) / 18\right), \\
\beta_{h}=\frac{\Phi(T)}{1-\exp \left(-\left(V_{i j}+35\right) / 10\right)}, \\
\alpha_{n}=\frac{0.07 \Phi(T) \exp \left(-\left(V_{i j}+65\right) / 20\right),}{1-\exp \left(-\left(V_{i j}+55\right) / 10\right)}, \\
\beta_{n}=0.125 \Phi(T) \exp \left(-\left(V_{i j}+65\right) / 80\right), \\
\phi(T)=3^{\left(T-6.3^{\circ} \mathrm{C}\right) / 10^{\circ} \mathrm{C}} .
\end{gathered}
$$

Here the variable $V_{i, j}$ describes the membrane potential of the neuron in site $(i, j)$ and the subscripts $(i, j)$ indicate the site of the neuron. $m, n$ and $h$ are parameters for the gate channel, and the capacitance of the membrane is $C_{m}=1$ $\mu \mathrm{F} / \mathrm{cm}^{2} . D$ is the intensity of coupling, $\varepsilon_{k l i j}$ describes the connection state (on or off) between site $(k, l)$ and site $(i, j)$, $i$ and $j$ are integers, $\varepsilon_{k l i j}=1$ if site $(k, l)$ is connected with site $(i, j)$ and $\varepsilon_{k l i j}=0$ otherwise. Clearly, if the fraction of randomly introduced shortcuts (i.e. rewired links) $p$ (probability) equals zero, $\varepsilon_{k l i j}=0$ only if site $(k, l)$ is one of the four nearest neighbors of site $(i, j)$. The maximal conductance of potassium is $\tilde{g}_{\mathrm{K}}=36 \mathrm{mS} / \mathrm{cm}^{2}$, the maximal conductance of sodium is $\tilde{g}_{\mathrm{Na}}=120 \mathrm{mS} / \mathrm{cm}^{2}$, the conductance of leakage current is $\tilde{g}_{\mathrm{L}}=0.3 \mathrm{mS} / \mathrm{cm}^{2}$ and the external injection current $I_{i j}=0$. The reversal potential $V_{\mathrm{K}}=-77 \mathrm{mV}$, $V_{\mathrm{Na}}=50 \mathrm{mV}$ and $V_{\mathrm{L}}=-54.4 \mathrm{mV} . \xi_{m}(t), \xi_{h}(t)$ and $\xi_{n}(t)$ are independent Gaussian white noise, and the statistical properties [33] of the channel noise are defined as follows:

$$
\begin{aligned}
& <\xi_{m}(t)>=0 ; \\
& \begin{aligned}
<\xi_{m}(t) \xi_{m}\left(t^{\prime}\right)>= & 2 \alpha_{m} \beta_{m} \delta\left(t-t^{\prime}\right) /\left[N_{\mathrm{K}}\left(\alpha_{m}+\beta_{m}\right)\right] \\
& =D_{m} \delta\left(t-t^{\prime}\right) .
\end{aligned} \\
& <\xi_{n}(t)>=0 ; \\
& <\xi_{n}(t) \xi_{n}\left(t^{\prime}\right)>=2 \alpha_{n} \beta_{n} \delta\left(t-t^{\prime}\right) /\left[N_{\mathrm{Na}}\left(\alpha_{n}+\beta_{n}\right)\right] \\
& \quad=D_{n} \delta\left(t-t^{\prime}\right) .
\end{aligned}
$$


Here, $D_{m}, D_{n}$ and $D_{h}$ describe the intensity of noise, function $\delta\left(t-t^{\prime}\right)=1$ at $t=t^{\prime}$ and $\delta\left(t-t^{\prime}\right)=0$ at $t \neq t^{\prime}$, and $N_{\mathrm{Na}}$ and $N_{\mathrm{K}}$ are the total numbers of sodium and potassium channels present in a given patch of the membrane, respectively. In the case of homogeneous ion channel density, $\rho_{\mathrm{Na}}=60$ $\mu \mathrm{m}^{-2}$ and $\rho_{\mathrm{K}}=18 \mu \mathrm{m}^{-2}$, the total channel number is decided by $N_{\mathrm{Na}}=\rho_{\mathrm{Na}} \cdot s$ and $N_{\mathrm{K}}=\rho_{\mathrm{K}} \cdot s$, and $s$ describes the membrane patch. Using mean-field theory, a statistical variable $[5,34,35]$ is defined to study the collective behaviors and statistical properties.

$$
\begin{gathered}
\left.F=\frac{1}{N^{2}} \sum_{j=1}^{N} \sum_{i=1}^{N} V_{i j}=<V\right\rangle_{s}, \\
R=\frac{<F^{2}>-<F>^{2}}{\frac{1}{N^{2}} \sum_{j=1}^{N} \sum_{i=1}^{N}\left(<V_{i j}^{2}>-<V_{i j}>^{2}\right)} .
\end{gathered}
$$

Here $R$ is a factor of synchronization, $N^{2}$ is the number of neurons and $V_{i j}$ is the membrane potential of the neuron. It is necessary to define the statistical variable $R$ to characterize the system's normalized variation and thus synchronization. $R$ may not be suitable to characterize the synchronization of the pattern of spiral waves, while it could be useful in detecting the critical bifurcation parameter inducing breakup or elimination of the spiral wave in networks of neurons as previously mentioned. As previously mentioned $[34,35]$, the curve of the factor of synchronization $v$ s. bifurcation parameter illustrates the phase transition of the spiral wave through points of sudden change. In [34], the author of the present work reported the additive Gaussian-colored noise-induced breakup in a regular network of H-R neurons, and multiplicative noise in the development of a spiral wave in regular networks of neurons (H-R, H-H) has also been investigated in detail [35]. Further numerical results have confirmed that a spiral wave can develop in networks (regular or small-world type) of neurons even if there is no external forcing current. The following section presents a numerical investigation of the robustness and phase of spiral waves in the small-world networks of $\mathrm{H}-\mathrm{H}$ neurons in the presence of channel noise where there are no external forcing currents acting on neurons.

\section{Numerical results and discussion}

The numerical studies have a time step $h=0.001$, external forcing current $I_{i j}=0,40000$ neurons in a two-dimensional array of $200 \times 200$ sites, and a no-flux boundary condition. The small-world connection network can be described by local regular networks (complete nearest-neighbor connections) and a long-range connection (shortcut) with a certain probability $p$. First, the case of no channel noise is considered, and the snapshots of the membrane potentials of neurons under different probabilities $(p=0.02,0.03,0.04$ and 0.05 ) are plotted with a transient period of about 500 time units.

The numerical results presented in Figure 1 show that a stable rotating spiral wave can develop completely with appropriate long-range probability, and no regular spiral wave is generated when the long-range probability exceeds a certain threshold. Note that the patterns in the figure are transient snapshots at $t=500$ time units, and the shape and contour of a stable spiral wave often remain unchanged as
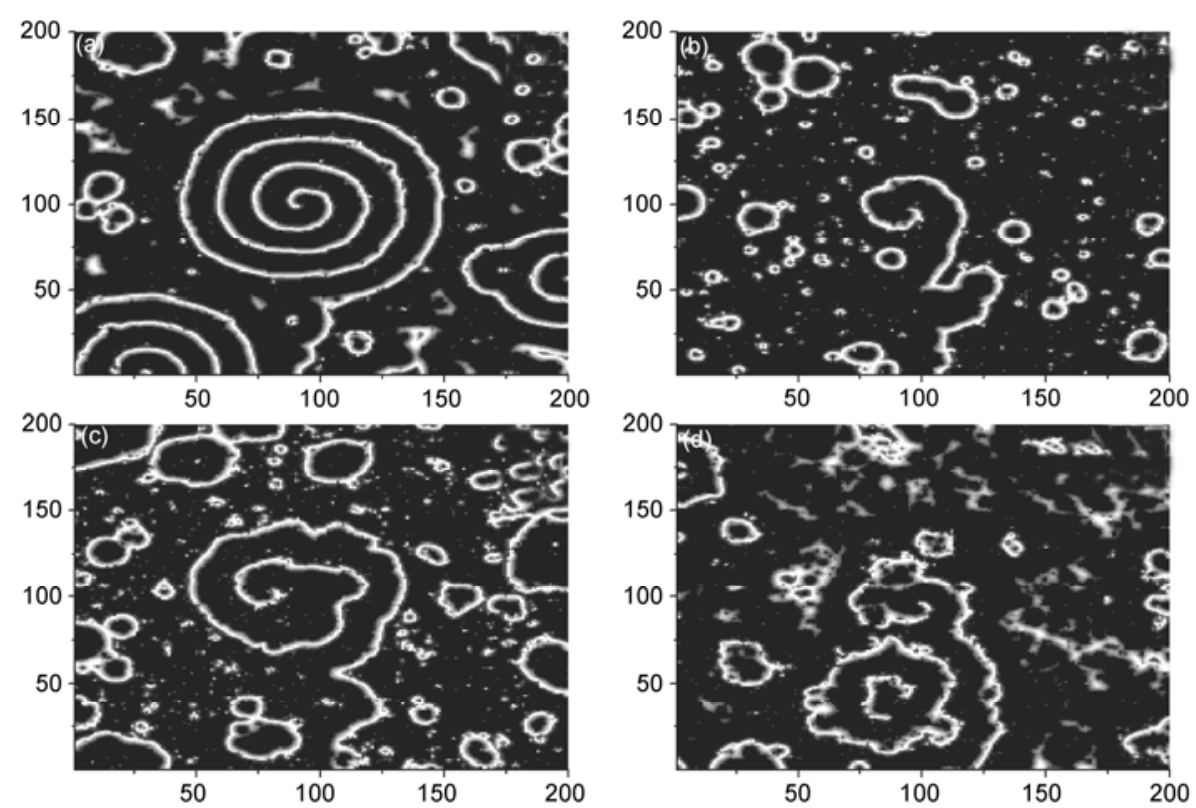

Figure 1 Spatiotemporal patterns developed within a transient period of about 500 time units for long-range probability $p=0.02$ (a), 0.03 (b), 0.04 (c) and 0.05 (d). The snapshots are plotted in grayscale from black (about $-80 \mathrm{mV}$ ) to white (about $-40 \mathrm{mV}$ ) and the coupling coefficient $D=1$. 
new segments of the spiral wave emerge for broken waves. The transient snapshots for any fixed duration show the distribution of membrane potentials of neurons, and stable spiral waves are maintained even though the membrane potential of a neuron at a site in the network varies with time. The corresponding factor of synchronization $R$ is given in Table 1.

It is found that a spiral wave can emerge and cover more area of the network if a lower long-range connection probability is used, and a smaller factor of synchronization is often employed. A smaller factor of synchronization also indicates a shorter transient period required to develop a spiral wave in a network. It is important to study the effect of channel noise on the phase transition of a spiral wave. Figure 2 illustrates the correlation of the synchronization factor and the membrane patch, which describes the intensity of channel noise, and the snapshots of membrane potentials of neurons for different fixed membrane patches (intensities of channel noise).

The results in Figure 2(b) confirm that the spiral wave breaks up when the intensity of channel noise increases to a certain threshold. A spiral wave emerges and covers a greater area in the case of weak channel noise, as seen by comparing the results in Figure 2(b) with those in Figure 1(a). The curve in Figure 2(a) shows that the factor of synchronization decreases with increasing intensity of the channel noise (smaller membrane being used). There are two distinct peaks ( $s=15$ and 20) in the curve in Figure 2(a), and the development of the spiral wave under channel noise close to the two peaks is investigated by checking the growth rate of a spiral wave in the networks. Figure 2(c) confirms that a longer transient period is required for a spiral wave to emerge and cover a greater area of a network in the case that the membrane patch (channel noise) corresponds to the two peaks in the curve. A spiral wave can emerge and cover the entire system with low long-range connection probability [36]. It is interesting to check the active role of channel noise in supporting a spiral wave in a network of neurons. As illustrated in Figure 1(c), no regular and distinct spiral wave occupies the networks with longrange connection probability $p=0.04$. Channel noise is selected with different intensities to check the effect of channel noise on the formation of the spatiotemporal pattern.

The results in Figure 3 show that appropriate channel noise can induce and develop a spiral wave in the networks of neurons at a certain long-range connection probability although the channel noise often induces breakup of the spiral wave. Comparing the results presented in Figure 3(b) with those presented in Figure 1(c), it is seen that the spiral

Table 1 Factors of synchronization under different long-range probability

\begin{tabular}{cccccc}
\hline Parameter & \multicolumn{5}{c}{ Value } \\
\hline$p$ & 0.02 & 0.03 & 0.04 & 0.05 & 0.06 \\
$R$ & 0.091359 & 0.180047 & 0.192383 & 0.182009 & 0.246083 \\
\hline
\end{tabular}
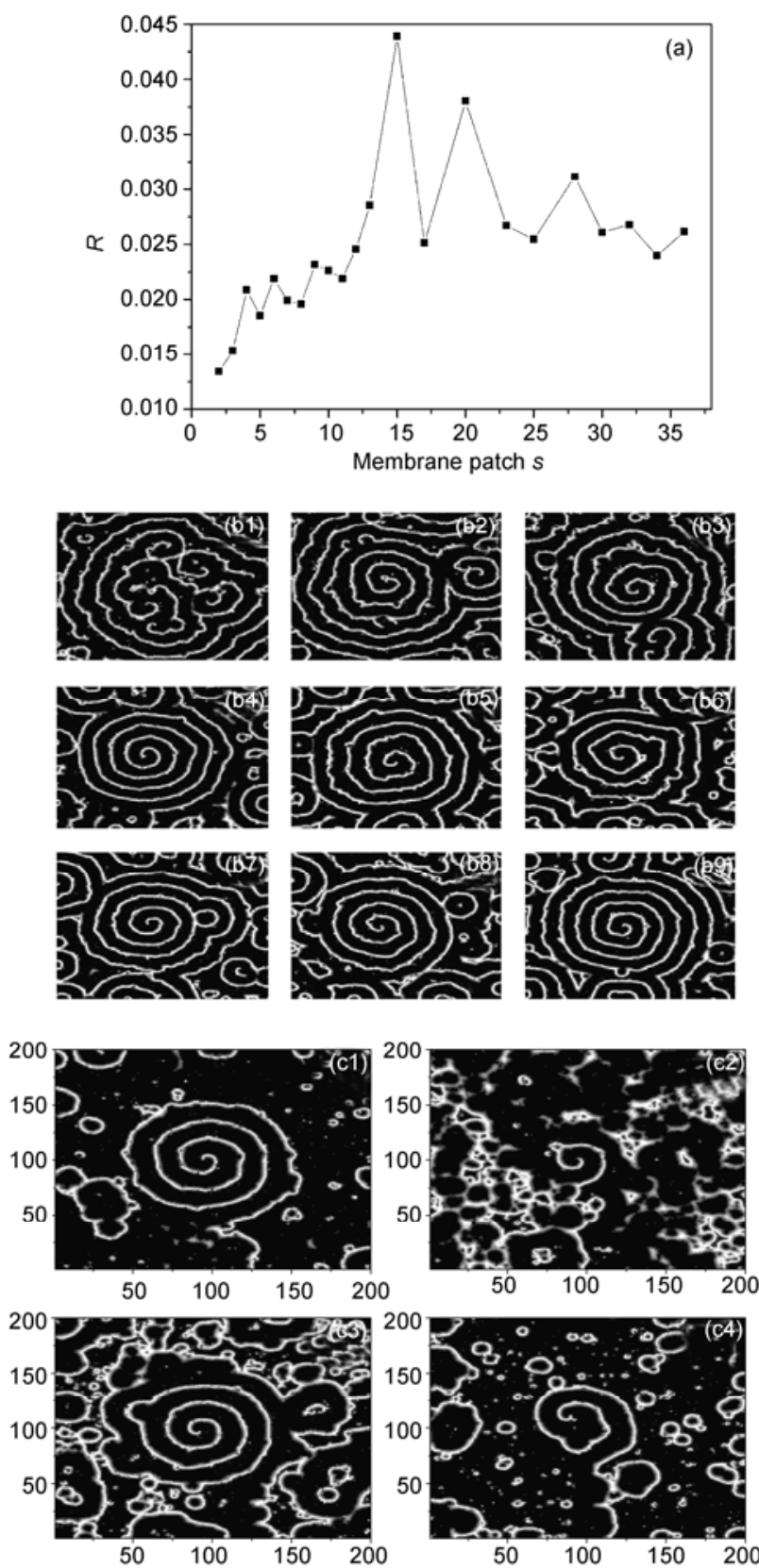

Figure 2 Calculated factor of synchronization $v s$. channel noise, described by the membrane patch (a) and spatiotemporal patterns developed within a transient period of about 2000 time units for $s=2$ (b1), 3 (b2), 4 (b3), 15 (b4), 17 (b5), 20 (b6), 28 (b7), 30 (b8) and 36 (b9). The snapshots of the development of a spiral wave are plotted for a transient period of about 200 time units for $s=13(\mathrm{c} 1), 15(\mathrm{c} 2), 17(\mathrm{c} 3)$ and $20(\mathrm{c} 4)$. The long-range probability is fixed at $p=0.02$, the coupling intensity is $D=1$ and the membrane temperature $T=6.3^{\circ} \mathrm{C}$. The snapshots are plotted in grayscale from black (about $-80 \mathrm{mV}$ ) to white (about $-40 \mathrm{mV}$ ).

wave covers a greater area when appropriate channel noise is introduced into networks of neurons. Clearly, channel noise can optimize the order of the spatiotemporal pattern in a network, and the optimized intensity of channel noise is close to the peak of the curve of the factor of synchronization vs. membrane patch. As is well known, a high probability of 

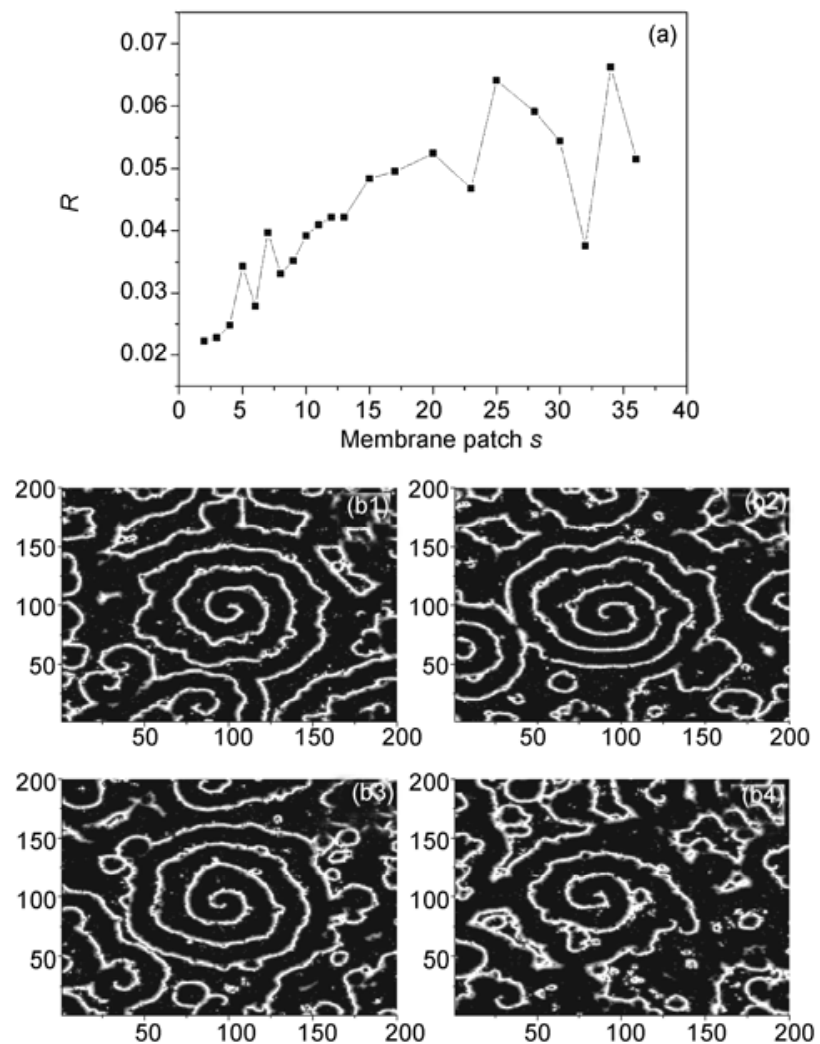

Figure 3 Calculated factor of synchronization vs. channel noise described by the membrane patch (a) and a spatiotemporal pattern developed within a transient period of about 2000 time units for $s=17$ (b1), 23 (b2), 25 (b3) and 28 (b4) at fixed long-range probability $p=0.04$, coupling intensity $D=1$, and membrane temperature $T=6.3^{\circ} \mathrm{C}$. The snapshots are plotted in grayscale from black (about $-80 \mathrm{mV}$ ) to white (about $-40 \mathrm{mV}$ ).

long-range connection and noise often destroy the order of the spatiotemporal pattern and break up the spiral wave. An ordered state can be generated when appropriate channel noise is introduced into media with small-world connections. It is the channel noise that optimizes the order of small-world networks, although it can also destroy the order. The membrane temperature often has an important role in determining the dynamics of neurons. Therefore, it is interesting to study the collective behaviors of spiral waves in networks with small-world connections. Figure 4 gives the factors of synchronization at different membrane temperatures and a fixed probability of a long-range connection.

The results in Figure 4 show that the factors of synchronization decrease with increasing membrane temperature and breakup of the spiral wave is induced in the small-world networks of $\mathrm{H}-\mathrm{H}$ neurons with fixed long-range probability $p=0.02$. It is the small-world effect that destroys the ordered state of networks, which differs from the case for regular networks, in which a certain high membrane temperature simply synchronizes all neurons with complete nearest-neighbor couplings (the media become homogeneous at a certain membrane temperature). To make a distinct
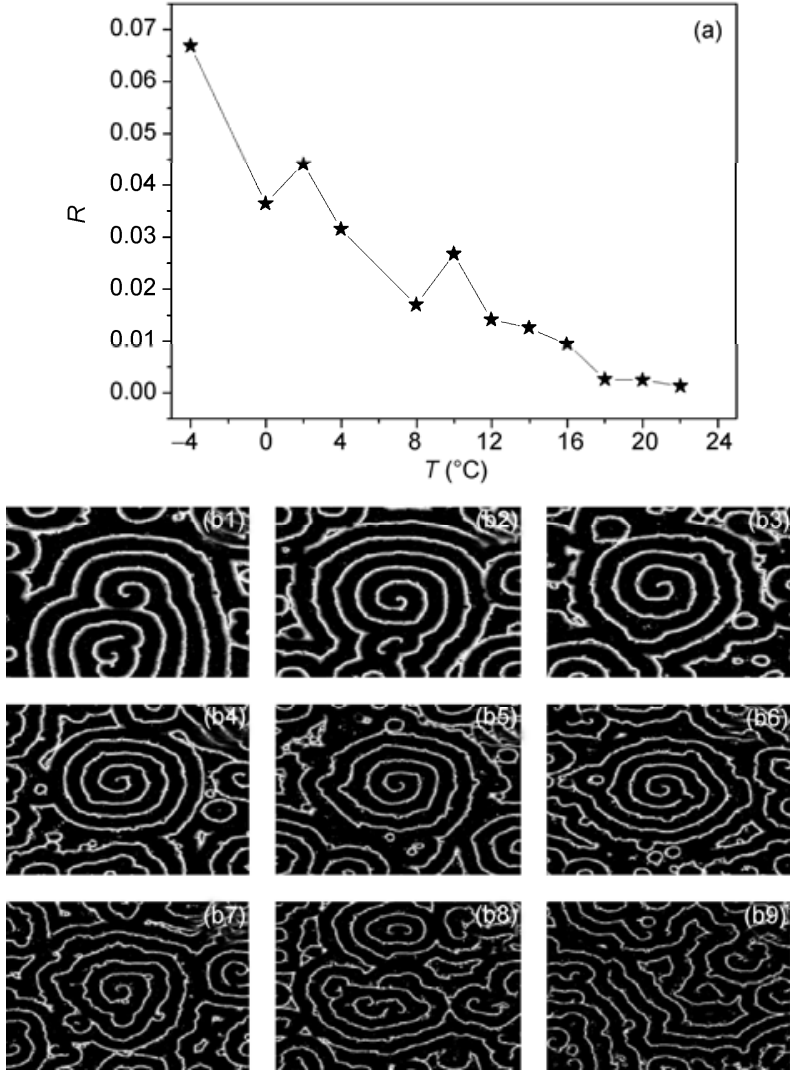

Figure 4 Calculated factor of synchronization $v s$. membrane temperature (a) and spatiotemporal patterns developed within a transient period of about 2000 time units for $T=-4^{\circ} \mathrm{C}$ (b1), $0^{\circ} \mathrm{C}$ (b2), $2^{\circ} \mathrm{C}(\mathrm{b} 3), 8^{\circ} \mathrm{C}$ (b4), $10^{\circ} \mathrm{C}$ (b5), $12^{\circ} \mathrm{C}(\mathrm{b} 6), 16^{\circ} \mathrm{C}(\mathrm{b} 7), 18^{\circ} \mathrm{C}$ (b8) and $20^{\circ} \mathrm{C}$ (b9) at fixed long-range probability $p=0.02$ and coupling intensity $D=1$. The snapshots are plotted in grayscale from black (about $-80 \mathrm{mV}$ ) to white (about $-40 \mathrm{mV})$.

comparison, the factors of synchronization for various membrane patches in regular networks are calculated and the results are shown in Figure 5.

The results in Figure 5 show that the factor of synchronization changes slowly with the membrane patch size, and there are no sudden changes in the curve of the synchronization factor $v s$. channel noise (membrane patch). This indicates that no phase transition occurs as the membrane patch size increases (decrease in the intensity of the channel noise), and a stable rotating spiral wave finally emerges to cover the network of neurons. On the other hand, breakup of the spiral wave is induced by increasing the intensity of channel noise (or decreasing the membrane patch size). These statements are confirmed by the snapshots of membrane potentials of neurons in the networks.

Comparing the results for the regular networks with those for small-world networks of neurons, it is found that a regular network actively supports the spiral wave and maintains its robustness against channel noise while the small-world network often induces the breakup of a spiral wave when the long-range connection probability exceeds a 

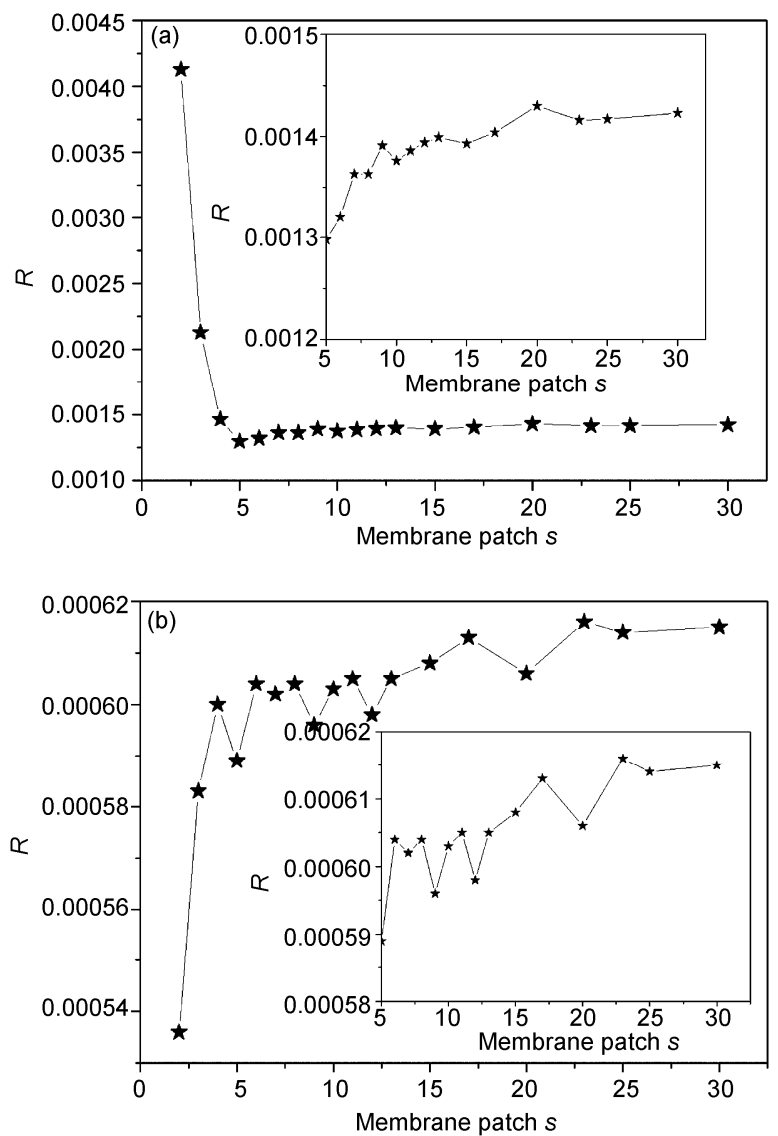

Figure 5 Calculated factor of synchronization vs. membrane patch (channel noise) within a transient period of about 2000 time units for membrane temperature $T=6.3^{\circ} \mathrm{C}$ (a) and $T=16.3^{\circ} \mathrm{C}$ (b). The inserted figures are enlarged illustrations for the membrane path $s \geqslant 5$.

certain threshold. Channel noise can play an active role in developing a spiral wave in small-world networks of $\mathrm{H}-\mathrm{H}$ neurons only when an appropriate intensity is selected. To date, most works have claimed that small-world connections better describe the complex connections of neurons tha regular networks, in which a neuron is only coupled with the four nearest adjacent neurons. To our knowledge, a regular connection supporting a spiral wave and long-range connections in small-world networks often destroys the spiral wave in homogeneous media. The reason could be that a regular connection results in regular variation in the potentials of the (five adjacent) neurons in the local domain owing to strong local coupling, and the long-range connection with high probability simply prevents neurons from changing simultaneously.

\section{Conclusions}

In this work, the channel noise-induced formation and changes in spiral waves in networks of $\mathrm{H}-\mathrm{H}$ neurons were investigated and some interesting results were found. A statistical variable referred to as the factor of synchronization was defined to measure the phase transition of the spiral wave. The small-world networks are described by the combination of local regular connection and long-range connenction with certain probability $p$. Long-range connections with high probability often prevent the formation of a spiral wave, and a generated spiral wave can cover a network of neurons only when appropriate intensity of the channel noise is selected. The corresponding curve of the factor of synchronization $v s$. channel noise (membrane patch) indicates coherent resonance-like behavior, odder selection and optimization with the channel noise. Breakup of the spiral wave in a small-world network occurs more easily than that in regular networks of neurons as the membrane temperature increases; that is, higher membrane temperature can induce breakup of the spiral wave more easily owing to the effect of small-world connections. The factor of synchronization changes slowly as the membrane patch size increases (or the intensity of channel noise decreases), and the spiral wave maintains its robustness against certain channel noise. As a result, selecting optimized channel noise is helpful in developing a stable spiral wave in the small-world networks of neurons through measuring and detecting the critical factor of synchronization $v s$. channel noise curve owing to its active role in propagating the electrical signal in the quiescent domain.

This work was partially supported by the National Natural Science Foundation of China (10747005 and 10972179) and the Natural Foundation of Lanzhou University of Technology (Q200706).

1 Zheng Z G, Feng X Q, Ao B, et al. Synchronization of groups of coupled oscillators with sparse connections. Europhys Lett, 2009, 87: 50006

2 Wang Q Y, Duan Z S, Perc M, et al. Synchronization transitions on small-world neuronal networks: Effects of information transmission delay and rewiring probability. Europhys Lett, 2008, 83: 50008

3 Weber S, Hütt M T, Porto M. Pattern formation and efficiency of reaction-diffusion processes on complex networks. Europhys Lett, 2008, 83: 28003

4 Brunnet L G. Multistability in networks of Hindmarsh-Rose neurons. Phys Rev E, 2008, 78: 061917

5 Wang C N, Ma J, Tang J, et al. Instability and death of spiral wave in a two-dimensional array of Hindmarsh-Rose neurons. Commun Theor Phys, 2010, 53: 382-388

6 Huang X Y, Troy W C, Yang Q, et al. Spiral waves in disinhibited mammalian neocortex. J Neurosci, 2004, 24: 9897-9902

7 Schiff S J, Huang X Y, Wu J Y. Dynamical evolution of spatiotemporal patterns in mammalian middle cortex. Phys Rev Lett, 2007, 98 : 178102

8 Perc M. Effects of small-world connectivity on noise-induced temporal and spatial order in neural media. Chaos Solitons Fractals, 2007, 31: 280-291

9 Perc M. Spatial decoherence induced by small-world connectivity in excitable media. New J Phys, 2005, 7: 252

10 Wang Q Y, Perc M, Duan Z S, et al. Delay-enhanced coherence of spiral waves in noisy Hodgkin-Huxley neuronal networks. Phys Lett A, 2008, 372: 5681-5687

11 Liu T, Li X, Liu X P. Integration of small world networks with multi-agent systems for simulating epidemic spatiotemporal trans- 
mission. Chinese Sci Bull, 2010, 55: 1285-1293

12 Zaikin A N, Zhabotinsky A M. Concentration wave propagation in two-dimensional liquid-phase self-oscillating system. Nature, 1970, 225: 535-537

13 Zhan M, Kapral R. Destruction of spiral waves in chaotic media. Phys Rev E, 2006, 73: 026224

14 Xiao J H, Hu G, Zhang $\mathrm{H}$, et al. Controlling the breakup of spiral waves in an excitable medium by applying time-delay feedback signals. Europhys Lett, 2005, 69: 29-35

15 García-Ojalvo J, Schimansky-Geier L. Noise-induced spiral dynamics in excitable media. Europhys Lett, 2005, 47: 298-303

16 Belmonte A, Flesselles J M, Ouyang Q. Spiral instability to line sources in forced chemical pattern turbulence. Europhys Lett, 1996, 35: $665-670$

17 Kessler D A, Levine H, Reynolds W N. Outer stability of spirals in excitable media. Europhys Lett, 1992, 19: 553-558

18 Coullet P, Lega J. Defect-mediated turbulence in wave patterns. Europhys Lett, 1998, 7: 511-516

19 Samie F H, Josem J. Mechanisms underlying ventricular tachycardia and its transition to ventricular fibrillation in the structurally normal heart. Cardiovasc, 2001, 50: 242-250

20 Yuan G Y, Wang G R, Chen S G. Control of spiral waves and spatiotemporal chaos by periodic perturbation near the boundary. Europhys Lett, 2005, 72: 908-914

21 Ramos J I. Dynamics of spiral waves in excitable media with local time-periodic modulation. Chaos Solitons Fractals, 2002, 13: 1383-1392

22 Chen J X, Hu B B. Spiral breakup and consequent patterns induced by strong polarized advective field. Europhys Lett, 2008, 84: 34002

23 Ma J, Jia J, Wang C N, et al. Instability of spiral wave induced by the deformation of the elastic excitable media. J Phys A, 2008, 41: 385105

24 Chen J X, Xu J R, Yuan X P, et al. Influences of periodic mechanical deformation on spiral breakup in excitable media. J Phys Chem B, 2009, 113: 849-853

25 Zhan M, Wang X G, Gong X F, et al. Phase synchronization of a pair of spiral wave. Phys Rev E, 2005, 71: 036212

26 Hou Z H, Xin H W. Noise-sustained spiral waves: Effect of spatial and temporal memory. Phys Rev Lett, 2002, 89: 280601

27 Hodgkin A L, Huxley A F. A quantitative description of membrane current and its application to conduction and excitation in nerve. $\mathrm{J}$ Physiol, 1952, 117: 500-544

28 Gosak M, Marhl M, Perc M. Pacemaker-guided noise-induced spatial periodicity in excitable media. Phys D, 2009, 238: 506-515

29 Ma J, Ying H P, Liu Y, et al. Development and transition of spiral wave in the coupled Hindmarsh-Rose neurons in two-dimensional space. Chin Phys B, 2009, 18: 98-105

$30 \mathrm{He} \mathrm{D} \mathrm{H,} \mathrm{Hu} \mathrm{G,} \mathrm{Zhan} \mathrm{M,} \mathrm{et} \mathrm{al.} \mathrm{Pattern} \mathrm{formation} \mathrm{of} \mathrm{spiral} \mathrm{waves} \mathrm{in} \mathrm{an}$ inhomogeneous medium with small-world connections. Phys Rev E, 2002, 65: 055204(R)

31 White J A, Rubinstein J T, Kay A R. Channel noise in neurons. Trends Neurosci, 2000, 23: 131-137

32 Schmid G, Goychuk I, Hänggi P. Capacitance fluctuations causing channel noise reduction in stochastic Hodgkin-Huxley systems. Phys Biol, 2006, 3: 248-254

33 Fox R F, Lu Y N. Emergent collective behavior in large numbers of globally coupled independently stochastic ion channels. Phys Rev E, 1994, 49: 3421-3431

34 Ma J, Jia Y, Tang J, et al. Breakup of spiral waves in the coupled Hindmarsh-Rose neurons. Chin Phys Lett, 2008, 25: 4325-4328

35 Ma J, Tang J, Zhang A H, et al. Robustness and breakup of spiral wave in a two-dimensional lattice networks of neurons. Sci ChinaPhys Mech Astron, 2010, 53: 672-679

36 Sinha S, Saraäki J, Kaski K. Emergence of self-sustained patterns in small-world excitable media. Phys Rev E, 2007, 76: 015101(R)

Open Access This article is distributed under the terms of the Creative Commons Attribution License which permits any use, distribution, and reproduction in any medium, provided the original author(s) and source are credited. 